\title{
Local hypertrichosis: A rare complication of a temporary henna tattoo
}

\author{
Nebahat Demet Akpolat ${ }^{1}$, Arzu Aras ${ }^{2}$ \\ ${ }^{1}$ Department of Dermatology, Beykoz State Hospital, ${ }^{2}$ Department of Pediatrics, Haydarpasa Numune Education and \\ Training Hospital, Istanbul, Turkey. E-mail: drdemetakpolat@gmail.com \\ Received: 20th November 2015, Revised: 18th April 2016, Accepted: 23rd May 2016
}

\begin{abstract}
SUMMARY: Akpolat ND, Aras A. Local hypertrichosis: A rare complication of temporary henna tattoo. Turk J Pediatr 2016; 58: 413-414.

Temporary henna tattoos have become increasingly widespread among children and young people, especially in holiday spots in recent years. Although reactions to henna tattoo are becoming progressively more common, only few cases of a henna pseudo-tattoo resulting in temporary hypertrichosis have been reported so far. Here, we have reported a 5-year-old girl who developed allergic contact dermatitis and localized hypertrichosis on her right arm after application of temporary henna tattoo during summer holiday.
\end{abstract}

Key words: Indian henna, temporary tattoo, localized hypertrichosis.

Temporary tattoo made by using Indian henna has become more popular among young people in recent years, in particular, in holiday resorts. Temporary tattoo, which is preferred because it is cheap, painless, easily applicable and disappears in a very short period of time, is reinforced by certain additives such as paraphenilendiamine to shorten the application time and to darken the color ${ }^{1,2}$. Paraphenilendiamine is a strong allergen and aggravates the complication risk that develops following the temporary tattoo. Among those complications that can develop are allergic contact dermatitis, scars, keloids, hypo-hyperpigmentation, urticaria, angioedema and anaphylaxis ${ }^{3}$. Localized hypertrichosis that develops following the temporary tattoos done using Indian henna is a rare complication reported in a few cases.

\section{Case Report}

A five-year- old girl was admitted because of a red blotch in her right arm and topical pilosity. It was learned that one and half months ago a temporary tattoo figuring a mermaid was processed by using the Indian henna; the tattoo automatically reversed back one month later but there were red blotches and hair increase in the same area; she did not apply to any healthcare institution and that no treatment was applied because of this complaint. The dermatological examination revealed that there were asymptomatic erythematous papule and hypertricosis in the area of mermaid figure in the right arm (Fig 1). Assuming that the localized hypertricosis developed due to Indian henna, it was noted that it would disappear within 3-4 months and so the patient was placed under clinical monitoring.

\section{Discussion}

Indian henna is applied to hair, skin and nails for religious, traditional and cosmetic reasons, in particular, in Arabic and Indian cultures for thousands of years 4,5 . Because of the recent use of additives that darken the tattoo colour and shorten the process time by adding it to the Indian henna, the frequency by which complications related to temporary tattoo have increased. Among these additives are, in particular, paraphenilendiamine, vinegar, olive oil, coal and clove ${ }^{1}$. It is considered that paraphenilendiamine is particularly responsible for the majority of the complications that occur following the temporary tattoo in which Indian henna is used. These complications mostly appear in the form of allergic contact dermatitis, hyper-hypopigmentation, scar, lichenoid reaction and keloid, and rarely, urticaria, erythema multiforme and angioedema ${ }^{3}$.

In our case, the hypertrichosis that developed 


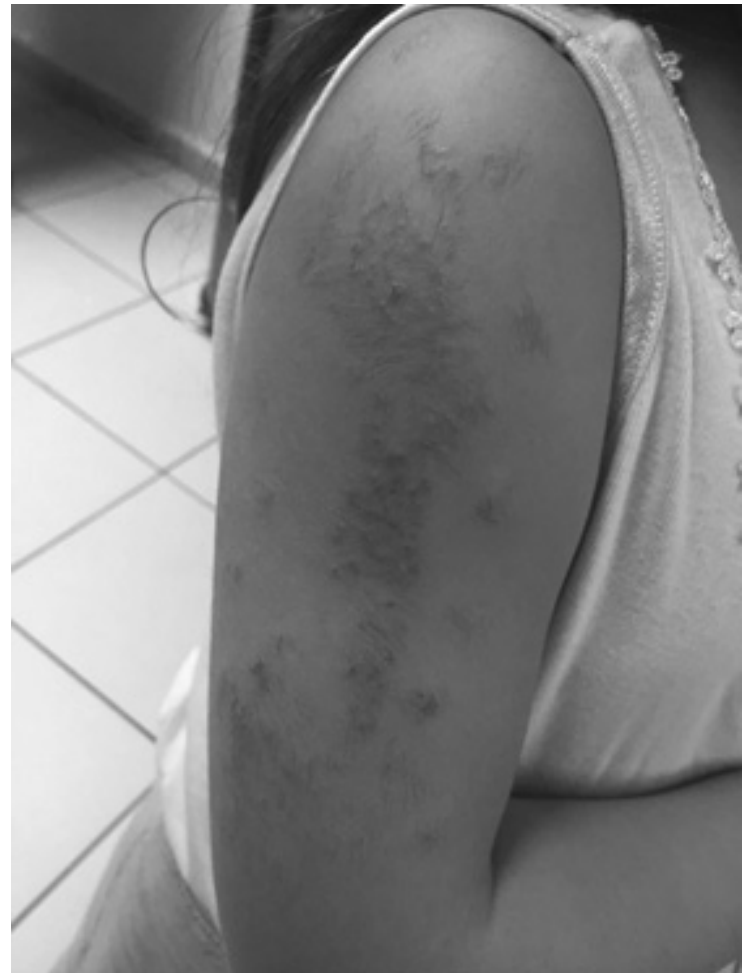

Fig.1. Asymtomatic erythematous papule and hypertricosis in the area of mermaid figure in the right arm.

in the tattoo zone was considered to have developed due to additives added to the Indian henna. Hypertrichosis that develops based on temporary tattoo made with Indian henna was diagnosed in only 8 people up until now ${ }^{2,3}$. Out of the reported cases, only 3 displayed hypertrichosis developed on the base of contact dermatitis area as is our case, $2,3,6$. It remains unknown whether there is a relation between contact dermatitis and hypertrichosis. In cases in the literature, hypertrichosis asymptomatically occurred 5-30 days after the tattoo and automatically healed back within 3-4 months ${ }^{2}$. Skin biopsy taken from one of the cases revealed an increase in velus hair follicle and a light peripheral fibrosis ${ }^{7}$.

Considering the fact that in our case and in other cases as well, the temporary tattoo was applied by street hawkers in summer months thus it is difficult to identify active ingredients and effect mechanism that could lead to hypertrichosis. Consequently, while the reason remains unknown, hypertrichosis is a rare complication that may develop based on the temporary tattoo and which spontaneously reverses back within 3 or 4 months. When this potential complication is known, the worries of the families may be settled and treatments with diagnose-oriented unnecessary invasive applications may be prevented.

\section{REFERENCES}

1. Ozcan D, Seckin D. A case of localized hypertrichosis due to temporary henna tattoo. Turk Dermatol 2014; 3: $164-165$

2. Habr C.E, Megarbane H. Temporary henna tattoos and hypertrichosis: a case report and review of the literature. J Dermatol Case Rep 2015; 2: 36-38.

3. Kaya Akış H, Çevirgen Cemil B, Canpolat F, Gönül M Hint kınası ile yapılan geçici dövmeye bağlı lokalize hipertrikoz: Bir olgu. Yeni Tip Dergisi 2014; 31: 205206.

4. Kind F, Scherer K, Bircher AJ. Contact dermatitis to para-phenylenediamine in hair dye following sensitization to black henna tattoos - an ongoing problem. J Dtsch Dermatol Ges 2012; 1057-1058.

5. Kazandijeva J, Grozdev I, Tsankov N. Temporary henna tattoos. Clin Dermatol 2007; 25: 383-387.

6. Kluger N, Garat H. Transient localized hypertrichosis on a temporary henna tattoo. Contact Dermatitis 2010; 62: 188-189.

7. del Boz J, Martin T, Samaniego E et al. Temporary localized hypertrichosis after henna pseudotattoo. Pediatr Dermatol 2008; 25: 274-275. 